\title{
Materiais Cristalinos Funcionais e Desenvolvimento Tecnológico
}

\author{
Agnaldo M. J. Junior, Igor L. de Andrade, Wesley F. Vaz, \\ Eduardo C. M. Faria \& Hamilton B. Napolitano
}

O estudo de novos materiais e o conhecimento estrutural da matéria é de grande importância para a ciência, neste trabalho é feita uma relação entre o estudo molecular, via difração de Raios X, através do método cristalográfico e o desenvolvimento tecnológico. É apresentado uma descrição dessa metodologia, amplamente aplicada para determinação estrutural de materiais sólido cristalinos. Assim, são demonstrados alguns exemplos de novos materiais resultantes do estudo da biodiversidade molecular.

Palavras-chave: Cristalografia; Novos materiais; Biodiversidade Molecular.

The study of new materials and the structural knowledge of the matter has great importance for science, in this work a relation is made between the molecular study, through diffraction of X-rays, through the crystallographic method and the technological development. A description of this methodology, widely applied for structural determination of crystalline solid materials, is presented. Thus, some examples of new materials resulting from the study of molecular biodiversity are demonstrated.

Keywords: Crystallography; New materials; Molecular Biodiversity. 


\section{Apresentação}

$\mathrm{O}$ avanço tecnológico e o desenvolvimento de novos materiais estão em uma nova era, impulsionada pela busca do bem-estar humano, demandando cada vez mais dos recursos naturais. Os estudos multidisciplinares são a chave para explicar fenômenos da natureza, estruturas de novos materiais e demais características necessárias aos avanços tecnológicos gerados pelo desenvolvimento de novos produtos e processos. Tais avanços exigem uma descrição minuciosa dos materiais e de suas moléculas. Dentre os diversos métodos e técnicas, o método cristalográfico destaca-se, no que tange à acurada descrição estrutural de um composto, devido à alta resolução com que se pode descrever a densidade eletrônica ${ }^{1,2}$.

A Cristalografia é o estudo dos cristais, de sua estrutura e propriedades físicas, assim o entendimento da estrutura interna dos materiais pode ser aplicado na otimização de propriedades mecânicas. Com isso, cristalógrafos têm participado no design e desenvolvimento de materiais, na metalurgia e na engenharia de cerâmica. Novas técnicas para investigar estruturas e defeitos de materiais estão sendo constantemente desenvolvidas usando métodos de difração de raios $\mathrm{X}$, nêutrons e elétrons. $\mathrm{O}$ trabalho dos cristalógrafos é crucial para projetar novos materiais, como os quasicristais e o grafeno, que surgem como materiais de promissora aplicabilidade no futuro próximo ${ }^{3}$.

O método é baseado no fenômeno da difração que ocorre devido à interação da radiação eletromagnética com a matéria cristalina, não necessitando conhecimento algum a respeito do composto a priori ${ }^{1}$. Seu principal objetivo é a determinação da disposição tridimensional dos átomos da estrutura molecular para compostos no estado cristalino, e com isso a construção da densidade eletrônica a partir das amplitudes e fases dos fatores de estrutura ${ }^{4}$. Sua importância na caracterização dos materiais e o entendimento de seu comportamento, quando aplicado por diferentes propósitos, se tornou de tão grande importância, que o ano de 2014 foi eleito, pela Organização das Nações Unidas , o ano Internacional da Cristalografia.

Há muito se reconhece que uma visão abrangente do sistema climático pode ser alcançada pela adoção de uma perspectiva termodinâmica. Duas abordagens principais podem ser visualizadas ao longo desta linha. Na primeira, o foco está nos mecanismos dinâmicos e processos físicos responsáveis pela transformação da energia de uma forma para outra. Na segunda abordagem, a ênfase recai na análise da irreversibilidade do sistema climático e, principalmente, da produção de entropia ${ }^{6}$. As mudanças climáticas e a redução da disponibilidade de combustíveis tem sido discutida por todo o mundo, demandando estudos multidisciplinares para o desenvolvimento de novas tecnologias, contribuindo para o avanço da engenharia7. Esta abordagem foi intensificada no século XXI, período marcado pela expansão tecnológica e sua utilização para modelagem estrutural e simulação de dados, incluindo neste contexto, desenvolvimento de novos materiais. Para o setor energético, as principais mudanças estão associadas a pesquisas de novos combustíveis, juntamente com itens correlacionados, incluindo os aditivos e componentes mecânicos que podem tornar viável o uso sustentável destes. Desta forma, o método cristalográfico, por meio da difração de raios $\mathrm{X}$ de monocristais e a difração de raios $\mathrm{X}$ pelo método do pó, representam uma das técnicas mais utilizadas para a determinação estrutural. Assim, todos os materiais cristalinos na natureza, se inorgânicos, orgânicos ou biológicos, macroscópicos ou microscópicos, tem suas próprias propriedades químicas e físicas, com forte dependência em sua estrutura atômica. ${ }^{8}$ Então, a cristalografia é de suma importância para descrever as funcionalidades dos materiais e seus componentes químicos.

\section{Cristalografia e Materiais Cristalinos Sólidos}

Um material cristalino pode ser classificado observandose a forma pela qual estão organizados seus átomos ou íons, uma característica desse tipo de material é que os átomos estão posicionados em um arranjo periódico ao longo das distâncias atômicas apresentando um agrupamento atômico ordenado que se repete tridimensionalmente. Em um cristal ideal, este arranjo em torno de um ponto específico deve ser igual ao arranjo em torno de qualquer ponto da rede cristalina?.

O trabalho de determinação da estrutura tridimensional, através da cristalografia de raios $\mathrm{X}$, envolve as seguintes etapas ${ }^{10}$ : 
(1) coleta de dados, (2) processamento dos dados, (3) resolução da estrutura, (4) refinamento, (5) validação e análise do modelo cristalográfico e (6) depósito no banco de dados apropriado. Essa técnica difratométrica, através da lei de Bragg ${ }^{11}$, fornece o conhecimento tridimensional da estrutura molecular - a nível atômico - para compostos no estado cristalino. A resolução da estrutura está associada à obtenção da fase $\varphi(\mathbf{h})$ para cada reflexão medida, de forma que seja possível a construção da densidade eletrônica $\rho(r)^{10}$ através da Equação 1:

$$
\rho(\boldsymbol{r})=\frac{1}{V} \sum_{h} F(\boldsymbol{h}) \exp i[-2 \pi \boldsymbol{h} * \boldsymbol{r}]=\frac{1}{V} \sum_{h}|F(\boldsymbol{h})| \exp i[-2 \pi \boldsymbol{h} * \boldsymbol{r}+\boldsymbol{\varphi ( h )}]
$$

onde $\mathrm{V}$ é o volume da cela unitária e $\mathrm{F}(\mathbf{h})$ é o fator de estrutura na forma complexa, sendo seu módulo $|F(\mathbf{h})|$ proporcional à intensidade da reflexão medida para direção h. A quantidade $\mathrm{h} \cdot \mathrm{r}$ corresponde ao produto escalar entre o vetor espalhamento no espaço recíproco $\mathrm{h}$ e o vetor posição no espaço direto $\mathrm{r}$. O problema da fase fica evidente através da Eq. (1), uma vez que o conhecimento da função $\rho(r)$ para cada posição $r$ da cela unitária depende do conhecimento das fases $\varphi(h)$. Sua solução para a cristalografia de pequenas moléculas em geral é obtida através dos Métodos Diretos ${ }^{9}$ e/ou do Método de Patterson'. As posições atômicas correspondem aos pontos de máximo da densidade eletrônica $\rho(r)$.

O refinamento é o procedimento de minimização da discordância entre os módulos dos fatores de estrutura observado $|\mathrm{F}(\mathbf{h})|$ obs e calculado $|\mathrm{F}(\mathbf{h})|$ cal, realizando pequenas modificações nos parâmetros atômicos a nos parâmetros atômicos [portanto das fases $\varphi(h)$ calculadas] determinados para a estrutura aproximada. Através da síntese de Fourier ${ }^{10}$, átomos podem ser acrescidos e ter seus parâmetros também refinados. O princípio utilizado é o dos Mínimos Quadrados ${ }^{11}$ em que a soma dos erros ao quadrado é minimizada através da função $M$ dada por:

$$
M=\sum_{\mathbf{h}} w_{\mathbf{h}}\left[\left.F(\mathbf{h})\right|_{o b s}-|F(\mathbf{h})|_{c a l}\right]^{2}
$$

onde w é o peso atribuído à cada reflexão, inversamente proporcional ao desvio padrão $\sigma_{\mathrm{h}}$ que está associado à medida da reflexão $h$.

O refinamento é um processo interativo na construção do modelo cristalográfico, guiado pela convergência cristalográfica dos dados associada à consistência química do modelo obtido. Caso esses dois critérios venham a divergir, prevalece a convergência cristalográfica levando a modificação do modelo químico inicialmente esperado. Em geral, Os programas mais comuns utilizados para resolução da estrutura são ${ }^{4}$ : SHELXS ${ }^{12}, \operatorname{SIR}^{13}$ e/ou DIRDIF ${ }^{14}$, e os programas mais utilizados para refinamento são: $\operatorname{SHELXL}^{12}$ e/ou $\operatorname{SIR}^{13}$. Finalizado o trabalho de construção do modelo estrutural, todas as informações cristalográficas podem ser armazenadas num arquivo padrão denominado CIF (Crystallographic Information File) $)^{15}$, adotado em 1991 pela IUCr (International Union of Crystallography). Esse formato de arquivo armazena cada aspecto da informação cristalográfica através de um código para o item (ex: _symmetry_cell_setting) seguido do respectivo valor ou resultado (ex: monoclinic), ambos no formato ASCII. Dessa forma, o intercâmbio das informações cristalográficas é viabilizado e, embora seja possível construir esse arquivo utilizando algum editor de texto, ele é usualmente gerado como uma função particular dentro do conjunto de programas utilizados em cristalografia. Cabe destacar que o formato CIF pode ser utilizado como fonte de informação estrutura de um composto em estudos de Química Teórica.

As estruturas cristalinas apresentaram diferentes conformações moleculares. No entanto, podem serobservados entre estruturas de uma mesma molecular, pequenas diferenças estruturais, afetar drasticamente o empacotamento observado no estados sólidos. Adicionalmente, distintas aplicações dessas moléculas podem ser observadas dependendo da formação de diferentes arranjos supramoleculares ${ }^{16}$.

As estruturas supramoleculares estão relacionadas às moléculas da mesma maneira que as estruturas moleculares estão relacionadas aos átomos. Embora os átomos desempenhem um papel crítico nos paradigmas da química, é a estrutura molecular que geralmente está no centro da química de muitos materiais tecnológicos e biológicos. Por relações de vizinhança em química supramolecular 
queremos dizer que duas moléculas estão próximas umas das outras durante um certo período. Durante este período de tempo, as duas moléculas podem ser consideradas ligadas independentemente da natureza da ligação e da razão pela qual os átomos estão próximos um do outro e desse fenômeno surge a relação estrutura-atividade (ambiental, biológica, etc) de um composto ${ }^{17}$.

A superfície de Hirshfeld (HS) ${ }^{18}$ é utilizada para obtenção de informações relacionadas aos contatos intermoleculares de um composto (estruturas supramoleculares), sua construção é baseada na densidade eletrônica de uma molécula em uma estrutura cristalina ${ }^{19,20}$. O estudo de interações moleculares através de HS auxilia no entendimento do arranjo molecular de um composto uma vez que permite analisar as ligações de hidrogênio clássica $(\mathrm{O}-\mathrm{H} \cdots \mathrm{N}, \mathrm{O}-\mathrm{H} \cdots \mathrm{O}$ e $\mathrm{N}-\mathrm{H} \cdots \mathrm{O})$ ou não clássicas $(\mathrm{C}-\mathrm{H} \cdots \mathrm{O})$ por meio de mapeamentos $(\mathrm{de}$, di e dnorm $)^{19,21}$. A teoria descrita por Hirshfeld ${ }^{18}$ define uma função peso para cada átomo em uma molécula. Em analogia a isso define-se uma nova função peso para uma molécula em um cristal:

$$
w(\mathbf{r})=\frac{\sum_{a \in \text { molecule }} \rho_{a}(\mathbf{r})}{\sum_{a \in c y s s t a l} \rho_{a}(\mathbf{r})}=\frac{\rho_{\text {promolecule }}(\mathbf{r})}{\rho_{\text {procystal }}(\mathbf{r})}
$$

onde o numerador é a soma das densidades eletrônicas médias dos átomos de uma molécula e o denominador é a soma das densidades eletrônicas dos átomos da amostra cristalina. Podemos ainda definir w(r) como:

$$
w(\boldsymbol{r}) \cong \frac{\rho_{\text {molecule }}(\boldsymbol{r})}{\rho_{\text {crystal }}(\boldsymbol{r})}
$$

Desta maneira, a forma da superfície será dada pela distribuição eletrônica das moléculas no espaço ${ }^{19}$. As HS podem ser geradas em três tipos de mapeamentos distintos, Figura 1.

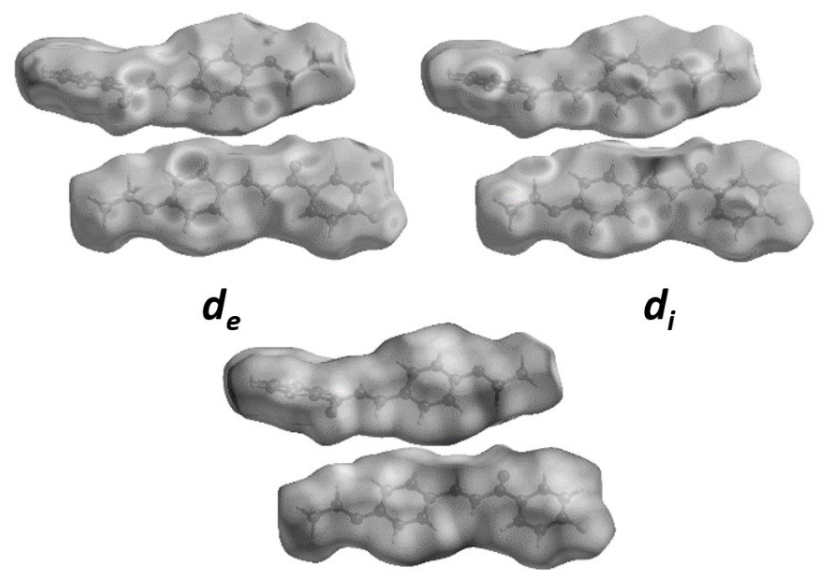

Normalizada

Figura 1. Representação da superfície de Hirshfeld apresentando diferentes funções mapeadas $\left(\mathrm{d}_{\mathrm{e}}, \mathrm{d}_{\mathrm{i}}\right.$ e normalizada) para uma estrutura cristalina com duas metóxi chalconas em sua unidade assimétrica ${ }^{22}$

A superfície $d_{e}$ representa a distância de uma molécula externa até a HS, já a superfície $d_{i}$ representa a distância da molécula interna até a HS, como mostrado na Figura 2. As regiões representadas em cores mais escuras indicam a presença de um contato intermolecular mais intenso.

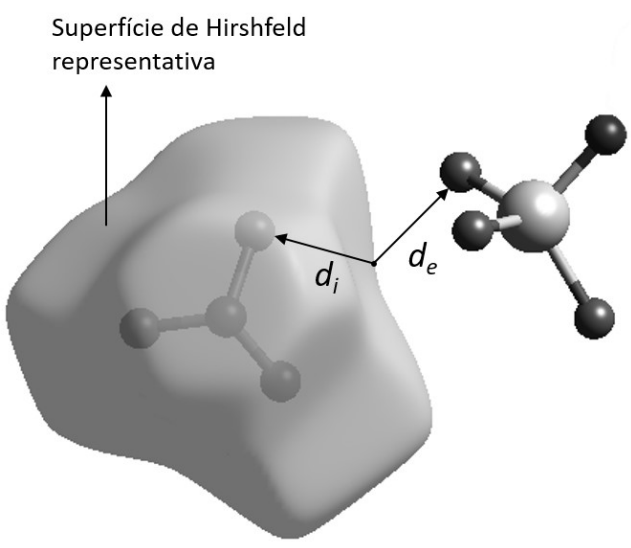

Figura 2. Representação das distâncias de e di na HS. 
Para a superfície $d_{e}$ essas regiões são caracterizadas como regiões receptoras de contato intermolecular. Por outro lado, na superfície $d_{i}$ as regiões mais escuras indicam locais em que a molécula atua como doador em um contato intermolecular ${ }^{19}$. O último tipo de mapeamento , HS normalizada (dnorm), é formado pela combinação das superfícies $d_{e}$ e $d_{i}$ representando todas as regiões de contatos intermoleculares mais intensos, independente se é receptor ou doador ${ }^{19}$. A Figura 3 representa outro tipo de análise de contatos intermoleculares, feito através de uma projeção bidimensional chamada de fingerprint (impressão digital). $\mathrm{O}$ objetivo é mapear todas os contatos da molécula em estudo, assim o gráfico bidimensional é gerado pela projeção de $\mathrm{d}_{\mathrm{e}}$ versus $\mathrm{d}_{\mathrm{i}}$, apresentando a contribuição percentual de cada tipo de interação presente ${ }^{23}$. O fingerprint também é capaz de evidenciar a presença ou ausência de um contato molecular específico, por exemplo, a ligação de hidrogênio classica $^{19}$.

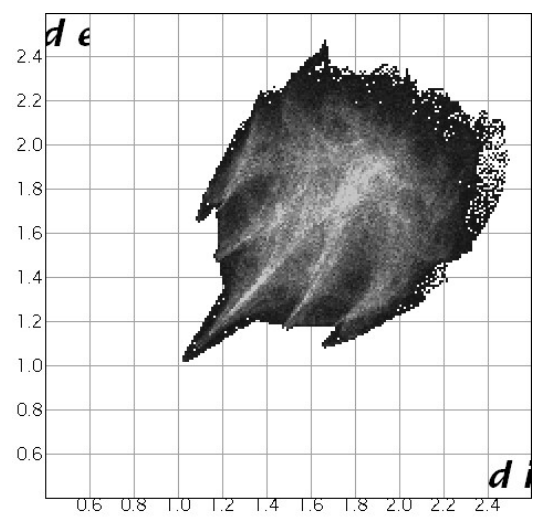

Figura 3:Fingerprint: representação bidimensional $\left(d_{e}\right.$ versus $\left.d_{i}\right)$ das interações presentes em uma molécula.

As HS aqui representadas são consolidadas na literatura. Temos ainda outros dois tipos de mapeamentos, curvada e indexada. A primeira permite analisar a planaridade de uma molécula enquanto que a segunda auxilia na identificação de interações tipo $(\pi \cdots \pi \text { e C-H } \cdots \pi)^{19}$.

\section{Materiais Cristalinos}

\section{INOVAÇÃO NA PERCEPTIVA AMBIENTAL}

A investigação dos processos ambientais através dos mecanismos da interação entre biodiversidade e ambiente, por meio da modelagem molecular, é uma metodologia capaz de ampliar o entendimento das propriedades dos compostos e direcionar a obtenção de novos produtos, em potencial, que tenham uma importância socioeconômica ${ }^{24}$. Dentro da biodiversidade, destaca-se a biodiversidade molecular, ou seja, diferentes moléculas de uma mesma classe. A biodiversidade molecular pode ser entendida em dois níveis: o químico e o estrutural, esse último o mais amplo ${ }^{25}$. Nessa investigação, o alinhamento entre as propriedades, na relação com a atividade, permite inferir sobre os produtos ambientais.

Um exemplo de compostos cristalinos aplicados a soluções voltadas ao meio ambiente que tem chamado atenção no meio científico, e que também tem sido objeto de estudo ${ }^{22,26-30}$ por parte do grupo de química teórica e estrutural de Anápolis (QTEA) são as chalconas. Quimicamente esses são compostos orgânicos que pertencem à família dos flavonóides, sendo abundantes na natureza e de fácil síntese em laboratório. Estruturalmente, elas apresentam uma cadeia aberta no qual dois anéis aromáticos estão unidos por um sistema carbonil $\alpha, \beta$-insaturado ${ }^{31}$.

Dentre as diversas aplicações das chalconas em processos e produtos ambientais, descritos na literatura, podemos destacar alguns trabalhos. Uma revisão foi feita ao abranger a fotoquímica e a atividade biológica de chalconas naturais mostrando o potencial da pesquisa com esses compostos nos campos da farmacobotânica, biotecnologia e estudos medicinais. ${ }^{31}$ Outro trabalho reportado mostra uma chalcona, que complexada ao íon $\mathrm{Fe}^{3+}$, demonstrou potencial de aplicação em processos ambientais visto que esse complexo permite a detecção seletiva de íons cianeto em sistemas aquosos, tais íons apresentam elevada toxicidade no meio ambiente. ${ }^{32}$ Também baseado em uma chalcona, foi sintetizado um sensor de fluorescência capaz de remover por completo, com auxílio de uma membrana 
de separação adequada, o íon $\mathrm{Fe}^{3+}$ de amostras de águas. ${ }^{33}$ Em paralelo, outro estudo com um agregado de chalcona, combinado com o íon $\mathrm{Al}^{3+}$, teve o intuito de desenvolver um método fluorimétrico para determinação desse íon em amostras de água ${ }^{34}$. Outro resultado obtido foi na sintetize de uma série de análogos de chalconas e alguns de seus derivados no intuito de estudar sua atividade larvicida frente o Culex quinquefasciatus (associado à elefantíase).$^{35}$ Além disso, foram realizados estudos de métodos analíticos para determinação de chalconas no estudo de fitotoxinas naturais em matrizes ambientais como água de superfícies, efluentes de tratamento de água, solo, etc. ${ }^{36}$ Outros estudos concluíram que a utilização de chalconas pode levar à descoberta de novos compostos nematicidas que apresentam uso potencial, como ingredientes ativos, no manejo de culturas. ${ }^{37}$ Contudo, estudos de docking molecular em estruturas fotoquímicas que possuíam alvos de proteínas do vírus da dengue e as chalconas se mostram como um dos grupos que mais fortemente se ligam nos alvos moleculares do vírus da dengue. ${ }^{38}$ Desta mesma forma, foram demonstrados eventuais novas aplicações de chalconas na proteção de cultivos e pesticidas orgânicos e para controle de plantas daninhas. ${ }^{39}$ Em vista disto, foram ultilizados estudos de análise quantitativa da relação estrutura-atividade em uma série de chalconas e esses mostraram que grupos substituintes retiradores de elétrons, no anel cetônico, geram bons agentes pesticidas ${ }^{40}$. A Figura 4 apresenta estruturas de chalconas e análogos de chalconas.

O crescimento da população mundial demanda por uma agricultura altamente produtiva. Dessa forma, desde a década de 50, novas tecnologias têm sido inseridas no processo de produção agrícola ${ }^{41-46}$. Estas envolvem, em sua maioria, o uso de ingredientes agroquímicos (IAQs) formulados como defensivos agrícolas (pesticidas, herbicidas, inseticidas, etc). Defensivos agrícolas de alta eficiência e mínimos impactos ambientais são desejáveis nas práticas agrícolas uma vez que quaisquer ameaças à produtividade têm impacto social e econômico ${ }^{42}$.

As investigações de novas formas sólidas, dentre elas a de IAQs, tem se intensificado nas discussões científicas
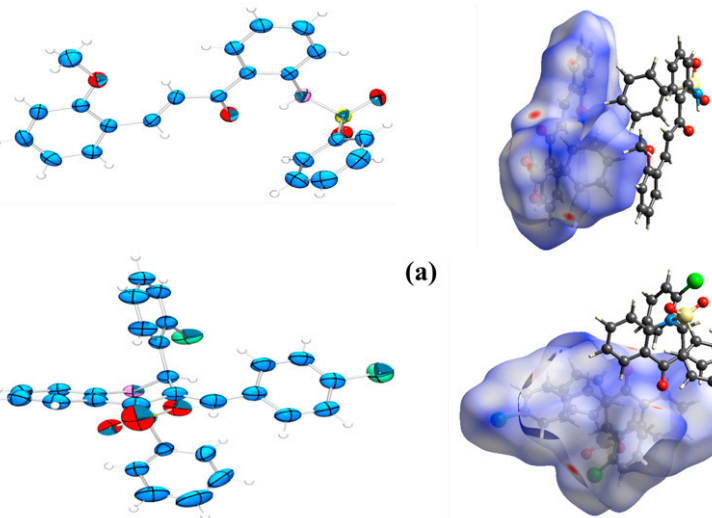

(a)

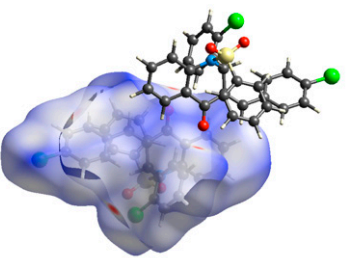

(b)

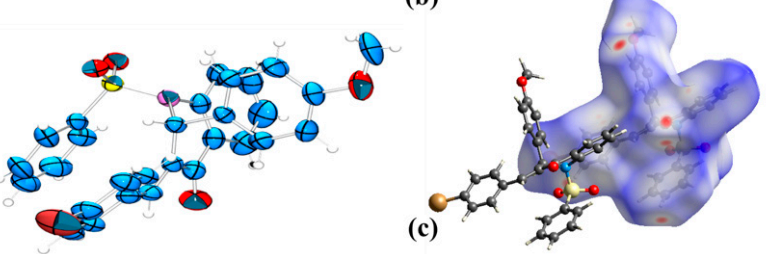

Figura 4. Três diferentes análogos de chalconas são representados por (a), (b) e (c). Elipsoides representados ao nível de probabilidade de $50 \%$ (esq) e superfícies de Hirshfeld normalizadas mostrando regiões de contatos intermoleculares (dir). Os átomos de H são mostrados como esferas de raio arbitrário.

constituindo-se como uma importante e promissora área de pesquisa ${ }^{47-50}$. Estes estudos se concentram na área de engenharia de cristais, que pode ser definida de forma objetiva como o design racional de sólidos moleculares funcionais, já, uma definição mais detalhada, aborda a engenharia de cristais como o entendimento das interações intermoleculares no contexto do empacotamento cristalino e na utilização de tal entendimento no design de novos sólidos com as propriedades quimicas desejadas ${ }^{51}$.

A otimização de propriedades físico-químicas de IAQs e o grande potencial na expansão e geração de propriedade intelectual de compostos ativos ${ }^{48,52,53}$ tem despertado o interesse de indústrias nesse campo de pesquisa uma vez que o planejamento de modificações cristalinas proporciona a redução nos custos do desenvolvimento de agentes agroquímicos, logo, resulta em impactos sobre o aspecto 
econômico, manufatura e comercialização destes ${ }^{54-56}$ e por possuirem propriedades fisico-químicas diferentes da forma convencional, elas constituem inovação tecnológica.

Em linhas gerais, o que se deseja é uma variação de propriedades que leve a compostos mais adequados para uma dada aplicação e isso pode ser obtido na diversidade de formas sólidas que um IAQ pode apresentar. A necessidade de otimização fica evidente quando problemas tais como a natureza amorfa de formas sólidas, a estabilidades/ solubilidade da formulação líquida, e transformação polimórfica são identificados ${ }^{57}$. A propriedade que determina a disponibilidade do defensivo agrícola para atuação no alvo é a solubilidade, além disso, normalmente, os defensivos disponíveis no mercado são agentes solúveis, porém, devido a processos de degradação, lixiviação e deriva, exibem uma limitada eficácia. Como consequência, estimula-se a aplicação demasiada e excessiva, resultando muitas vezes em problemas ambientais ${ }^{58}$.

Novas formas sólidas de herbicidas e pesticidas ainda são um campo relativamente inexplorado. Uma melhor compreensão das propriedades de estado sólido das novas e das formas existentes desses ingredientes ativos pode representar um poderoso acréscimo de know-how para a indústria e um desafio para os químicos de estado sólido, na investigação e na busca de novos cristais moleculares e múltiplas formas cristalinas. Na literatura podemos encontrar pesquisas que buscam desenvolver novas formas sólidas de diversos agroquímicos, sempre na perspectiva de obter compostos com propriedades otimizadas, mais amigáveis ao meio ambiente e. se possível, com potencial para novas aplicações. O primeiro exemplo que mostraremos é o do herbicida bentazona (2,2-dióxido de 3-isopropil(1H)benzo-2,1,3-triadizin-4-ona), pertencente ao grupo químico das benzotiadiazinonas, sendo um agroquímico da classe toxicológica I (extremamente tóxico).

Através de técnicas de cristalização em solução e diretamente no estado sólido, foram caracterizados dois novos polimorfos do Bentazon. ${ }^{59}$. A forma comercial desse herbicida é uma mistura de dois polimorfos e até a publicação de seu trabalho apenas a estrutura cristalina de um deles ${ }^{60}$ havia sido publicada. Em longo prazo, a utilização de bentazona pode causar efeitos danosos ao ambiente aquático por ser altamente móvel e persistente no meio ambiente ${ }^{61}$, estudo futuros poderão mostrar se essas novas formas sólidas são capazes de contornar ou minimizar esses efeitos. Uma oportunidade de pesquisa nessa área está na busca de novas formas sólidas do metomil, este é um inseticida da classe dos carbamatos que exibe atividade contra as principais pragas de Lepidoptera, Coleoptera, Diptera e Hemiptera, que afetam o algodão, soja, milho, frutas e vegetais.É muito tóxico para mamíferos sendo classificado pela Agência de Proteção Ambiental dos EUA como pesticida de uso restrito de Classe I.

Recentemente descobriu-se que a mais relevante praga do milho, a Spodoptera frugiperda, conhecida como lagarta-do-cartucho, tem apresentado resistência ao metomil, inseticida muito usado no combate ao problema. Segundo especialistas, a taxa de mortalidade das lagartas em estudo no estado do Mato Grosso ficou entre $15 \%$ e $30 \%$, o que é considerado um baixo índice de efetividade (50\% seria o ideal, uma vez que a morte de muito menos do que a metade, é indica de que há problema de resistência $)^{62}$. Até o momento são conhecidas quatro formas cristalinas do metomil ${ }^{63-65}$.

O herbicida metribuzin, [4-amino-6-tert-butil-3metiltio-1,2,4-triazina-5-(4H)-ona] pertence ao grupo das triazinas e, comercialmente, se apresenta em duas formas: pó molhável ou suspensão concentrada. Sua estrutura molecular cristalograficamente elucidada ${ }^{66}$ foi publicada em 1989. No Brasil, está registrado para controle de plantas daninhas mono e dicotiledôneas, sendo aplicado em pré e pós-emergência das plantas daninhas da cultura da cana-de-açúcar (entre outras) ${ }^{67}$. Uma problemática do metribuzin vem do fato de que doses elevadas em soja induzem aumentos na fitotoxicidade das plantas e, consequentemente, redução no vigor das sementes, o efeito residual de metribuzin aplicado em pré-emergência, causa menor potencial de armazenamento e pior desempenho da semente na fase de estabelecimento no campo $^{68}$. A obtenção de novas formas sólidas do metribuzin é apresentada em um estudo ${ }^{69}$ onde pesquisadores, utilizando variação na polaridade do solvente, identificaram que o 
metribuzin exibe comportamento polimórfico, cristalizando como placas e agulhas, gerando diferentes conjuntos moleculares resultantes de um delicado equilíbrio de forças intermoleculares fracas, dessa maneira, duas modificações polimórficas desse herbicida foram estruturalmente caracterizadas.

Por fim, apresentamos um estudo acerca do herbicida Picloram (ácido 4-amino 3,5,6 tricloro-2piridinacarboxílico), muito utilizado no controle de plantas daninhas dicotiledôneas arbustivas e arbóreas, em pósemergência e em pastagens. Esse herbicida pertence ao grupo dos mimetizadores de auxinas destacando-se pelo alto período de atividade residual no solo característica que impede o cultivo a curto prazo de várias espécies agrícolas não seletivas e também pelo alto risco de contaminação do lençol freático ${ }^{70}$. Embora sua estrutura cristalográfica ainda não tenha sido relatada, a do seu sal de guanidínio é conhecida ${ }^{71}$, além disso, recentemente foi publicada uma estrutura de um sal sódico do picloram ${ }^{72}$. Isso demonstra as muitas oportunidades de desenvolvimento e obtenção de novas formas sólidas que possam vir a resolver as diversas problemáticas envolvidas no uso de agroquímicos.

\section{INOVAÇÃO NA PERSPECTIVA ENERGÉTICA}

As mudanças climáticas têm estimulado estudos que geram novas políticas ambientais e consequentemente, novas tendências. Uma das principais preocupações é o nível de emissão atmosférica que vem crescendo. Como exemplo disso, de acordo com as projeções do Intergovernmental Panel on Climate Change, a partir do ano de 2015, a concentração de $\mathrm{CO}_{2}$ na atmosfera era 400 partes por milhão (ppm), enquanto é previsto que em 2040 aumente para $450 \mathrm{ppm}$, resultando no aumento da temperatura global em $2^{\circ} \mathrm{C} .{ }^{73}$ Como mecanismos paliativos, o governo incentiva o uso dos biocombustíveis e sistemas de tratamentos posteriores, por meio de sansões de lei e outros mecanismos políticos. Para a construção do estado de arte e identificação de todas as possibilidades relacionadas com conquistas ambientais, a cristalografia surge como uma ferramenta para a compreensão das moléculas e descrição de suas propriedades.
Entre estas alternativas tecnológicas de combustíveis, a base hidrogenada em células combustíveis vem sendo aplicada para geração de energia. O hidrogênio apresenta algumas vantagens em relação aos combustíveis convencionais, como por exemplo, energia contendo três vezes melhores que os outros. As células combustíveis são uma transformação eletroquímica que converte energia química em energia elétrica, combinando um átomo de oxigênio em dois átomos de hidrogênio e energia termal. Além disso, neste processo de geração de energia é produzido apenas água, na qual é benéfico ao meio ambiente. Entretanto, para aplicar este componente como combustível, é necessário estabilizar o mecanismo de segurança para armazenar e transportar. Por meio da Cristalografia componentes baseados em zinco e oxigênio tem sido caracterizado como tanques de combustíveis moleculares, considerando que eles têm a capacidade de separar hidrogênio em baixas pressões e temperaturas ambientes $^{74}$. Então, a Cristalografia pode ser aplicada para definir estruturas moleculares oferecendo subsidio por elucidação molecular e corresponder com aplicações tecnológicas.

\section{Conclusão}

O termo inovação engloba a introdução de algo novo. Inovações bem-sucedidas recuperam os custos para inventá-las e implementá-las, além de ser um prêmio para aqueles que estavam dispostos a assumir o risco de investir nelas. Tal definição vale apenas para inovações que possam ser aplicadas e, portanto, aceitas pelo mercado. No caso de fármacos, vitaminas e agroquímicos a inovação condicionase pela natureza dos organismos vivos. A engenharia de cristais visa melhorar e controlar as propriedades dos materiais de tais ingredientes biologicamente ativos e pode contribuir substancialmente para atender as inovações demandadas nos mais diversos setores da cadeia produtiva. Com a contribuição de cristalógrafos, foi possível então a elucidação estrutural de diversos princípios ativos de medicamentos e biomoléculas, estas de suma importância, tais como a Vitamina B12, a penicilina, a insulina e até o 
DNA, haja vista que são grandes exemplos de uma inovação bem aplicada.

Esta metodologia permite definir a composição da estrutura molecular relacionando-as com suas propriedades. É claro que a elucidação completa do método cristalográfico e a imensa gama de sua aplicação envolve diversos outros conceitos não abordados aqui, porém, fica evidente que sempre que se fala de inovação e tecnologia, é mérito destacar a importância do estudo dos materiais cristalinos.

\section{Referências Bibliográficas}

1. Drenth J. Principles of Protein X-Ray Crystallography. Springer New York; 2007.

2. Fernandes WB, Napolitano HB, Nodaperes C, Martins FT, Lariucci C. Aplicações Tecnológicas da Metodologia Cristalográfica. Rev Process Químicos. 2010.

3. Betts K. Crystallography: Understanding the Nature of Chemical Bonds and Molecular Structure.; 2015.

4. Olliveira Sallum L, Lúcio Benedito de Aquino G, Napolitano HB. Cristalografia de chalconas metoxiladas. In: Ciências Moleculares 2. Anápolis: Universidade Estadual de Goiás, Pró-Reitoria de Pesquisa e Pós-Graduação; 2012:458.

5. Guionneau P. Crystallography and spin-crossover. A view of breathing materials. Dalt Trans. 2014;43:382-393.

6. Lucarini V, Fraedrich K, Lunkeit F. Thermodynamics of climate change: generalized sensitivities. Atmos Chem Phys. 2010;10(20):9729-9737. doi:10.5194/acp-10-9729-2010

7. Zarghami E. New Technologies in Construction Materials Based on Environmental Approach (Case Study: Double Skin Facades). Mediterr J Soc Sci. 2015;6(6):17-24.

8. Li J, Junliang S. Application of X-ray Diffraction and Electron Crystallography for Solving Complex Structure Problems. Acc Chem Res. 2017;50:2737-2745.

9. Callister Jr. WD, Rethwish DG. A Estrutura dos Sólidos Cristalinos. In: Ciência e Engenharia de Materiais. 7a Edição. Rio de Janeiro; 2007.

10. Giacovazzo C. Fundamentals of Crystallography. Oxford University Press; 2002.

11. Viterbo D. Solution and refinement of crystal structures. In: Giacovazzo C, ed. Fundamentals of Crystallografhy. Oxford University Press; 2002.

12. Sheldrick GM. Crystal structure refinement with SHELXL. Acta Crystallogr Sect C. 2015;71(1):3-8. doi:10.1107/S2053229614024218

13. Burla MC, Caliandro R, Carrozzini B, et al. Crystal structure determination and refinement via SIR2014. J Appl Crystallogr. 2015;48(1):306-309. doi:10.1107/S1600576715001132

14. Sheldrick GM, C. K, Goggard. The DIRDIF program system. In: Crystallographic Computing. Vol. 3. ; 1985:216.

15. Cambridge Structural Database. Cambridge Crystallographic Data Centre. 2010.

16. Martins M, Meyer A, Salbego P, et al. Synthesis, Crystal Structure, and Supramolecular Understanding of 1,3,5-Tris(1-phenyl-1H-pyrazol-5-yl) benzenes. Molecules. 2017;23(1):22. doi:10.3390/molecules23010022

17. Turro NJ. Molecular structure as a blueprint for supramolecular structure chemistry in confined spaces. Proc Natl Acad Sci. 2005;102(31):1076610770. doi: 10.1073 pnas. 0501376102

18. Hirshfeld FL. Bonded-atom fragments for describing molecular charge densities. Theor Chim Acta. 1977;44(2):129-138.

19. McKinnon JJ, Spackman MA, Mitchell AS. Novel tools for visualizing and exploring intermolecular interactions in molecular crystals. Acta Crystallogr Sect B Struct Sci. 2004;60(6):627-668.

20. Spackman MA, Jayatilaka D. Hirshfeld surface analysis. CrystEngComm. 2009;11(1):19-32.

21. Desiraju GR, Steiner T. The Weak Hydrogen Bond: In Structural Chemistry and Biology. Vol 9. International Union of Crystal; 2001.

22. Carvalho PS, Custodio JMF, Vaz WF, et al. Conformation analysis of a novel fluorinated chalcone. J Mol Model. 2017;23(3):97. doi:10.1007/ s00894-017-3245-8

23. Spackman MA, McKinnon JJ. Fingerprinting intermolecular interactions in molecular crystals. CrystEngComm. 2002;4(66):378-392.

24. Yuriev E, Coote ML. Molecular Modelling: Advances in Biomolecular and Materials Modelling. Aust J Chem. 2011;64(7):885. doi:10.1071/ CH11232

25. Singh SB, Pelaez F. Biodiversity, chemical diversity and drug discovery. Prog Drug Res. 2008;65:142-174. doi:10.1007/978-3-7643-8117-2_4

26. Custodio JMF, Santos FG, Vaz WF, et al. Molecular structure of hybrid imino-chalcone in the solid state: X-ray diffraction, spectroscopy study and third-order nonlinear optical properties. J Mol Struct. 2018;1157:210-221. doi:10.1016/j.molstruc.2017.12.023

27. Custodio J, Michelini L, Castro M, et al. Structural Insights on a Novel Anticancer Sulfonamide Chalcone. New J Chem. 2018. doi:10.1039/ C7NJ03523C

28. Custodio JMF, Vaz WF, de Andrade FM, Camargo AJ, Oliveira GR, Napolitano HB. Substitution effect on a hydroxylated chalcone: Conformational, topological and theoretical studies. J Mol Struct. 2017;1136:69-79. doi:10.1016/j.molstruc.2017.01.076

29. Custodio J, Faria E, Sallum L, et al. The Influence of Methoxy and Ethoxy Groups on Supramolecular Arrangement of Two 
Methoxy-chalcones. J Braz Chem Soc. 2017;28(11):2180-2191. doi:10.21577/0103-5053.20170067

30. Custodio J, Moreira C, Valverde C, de Aquino G, Baseia B, Napolitano H. Hirshfeld Surfaces and Nonlinear Optics on Two Conformers of a Heterocyclic Chalcone. J Braz Chem Soc. 2017;29(2):168-179. doi:10.21577/0103-5053.20170136

31. Rozmer Z, Perjési P. Naturally occurring chalcones and their biological activities. Phytochem Rev. 2014;15(1):87-120. doi:10.1007/s11101-0149387-8

32. Yang $\mathrm{W}$, Cheng $\mathrm{Z}, \mathrm{Xu} \mathrm{Y}$, et al. A highly selective fluorescent chemosensor for cyanide anions based on a chalcone derivative in the presence of iron(III) ions, and its capacity for living cell imaging in mixed aqueous systems. New J Chem. 2015;39(9):7488-7494. doi:10.1039/C5NJ01043H

33. Velmurugan K, Prabhu J, Tang L, et al. A simple chalcone-based fluorescent chemosensor for the detection and removal of $\mathrm{Fe} 3+$ ions using a membrane separation method. Anal Methods. 2014;6(9):2883. doi:10.1039/c3ay42139b

34. Mahajan PG, Bhopate DP, Kolekar GB, Shivajirao R. Patil. A Chalcone Based Novel Fluorescent Nanoprobe for Selective Detection of Al3+ Ion in Aqueous Medium. J Lumin Appl. 2015;2(1):1-13. doi:10.7726/ jla.2015.1001

35. Begum NA, Roy N, Laskar RA, Roy K. Mosquito larvicidal studies of some chalcone analogues and their derived products: Structure-activity relationship analysis. Med Chem Res. 2011;20(2):184-191. doi:10.1007/ s00044-010-9305-6

36. Hoerger CC, Schenzel J, Strobel BW, Bucheli TD. Analysis of selected phytotoxins and mycotoxins in environmental samples. Anal Bioanal Chem. 2009;395(5):1261-1289. doi:10.1007/s00216-009-3088-y

37. Caboni P, Aissani N, Demurtas M, Ntalli N, Onnis V. Nematicidal activity of acetophenones and chalcones against Meloidogyne incognita and structure-activity considerations. Pest Manag Sci. 2016;72(1):125130. doi:10.1002/ps.3978

38. Powers C, Setzer W. An In-Silico Investigation of Phytochemicals as Antiviral Agents Against Dengue Fever. Comb Chem High Throughput Screen. 2016; 19(7):516-536. doi:10.2174/138620731966616050612371 5

39. Díaz-Tielas C, Graña E, Reigosa MJ, Sánchez-Morreiras AM. BIOLOGICAL ACTIVITIES AND NOVEL APPLICATIONS OF CHALCONES. Planta Daninha. 2016;34(3):607-616. doi:10.1590/ s0100-83582016340300022

40. Kumar R, Sharma P, Shard A, Tewary DK, Nadda G, Sinha AK. Chalcones as promising pesticidal agents against diamondback moth (Plutella xylostella): Microwave-assisted synthesis and structure-activity relationship. Med Chem Res. 2012;21(6):922-931. doi:10.1007/s00044011-9602-8
41. Powles SB, Yu Q. Evolution in Action: Plants Resistant to Herbicides. Annu Rev Plant Biol. 2010;61(1):317-347. doi:10.1146/annurevarplant-042809-112119

42. Plimmer JR, Gammon DW, Ragsdale and NR. Encyclopedia of Agrochemicals. John Wiley Sons, Inc. 2003;17(1981):528-529-751785-843-1166-1199. doi:10.1002/047126363X

43. Schleifer KJ. Challenges in agrochemicals design. J Cheminform. 2013;5(1):O17. doi:10.1186/1758-2946-5-S1-O17

44. Delaney J, Clarke E, Hughes D, Rice M. Modern agrochemical research: a missed opportunity for drug discovery? Drug Discov Today. 2006;11(17-18):839-845. doi:10.1016/j.drudis.2006.07.002

45. David Tilman, Kenneth G. Cassman, Pamela A. Matson RN\& SP. Nature. Nature. 2002;418:671-677.

46. Tilman D, Fargione J, Wolff B, et al. Forecasting agriculturally driven global environmental change. Science (80- ). 2001;292(5515):281-284. doi:10.1126/science. 1057544

47. Jeschke $P$. The unique role of halogen substituents in the design of modern agrochemicals. Pest Manag Sci. 2010;66(1):10-27. doi:10.1002/ ps. 1829

48. Jeanmart S, Edmunds AJF, Lamberth C, Pouliot M. Synthetic approaches to the 2010-2014 new agrochemicals. Bioorganic Med Chem. 2016;24(3):317-341. doi:10.1016/j.bmc.2015.12.014

49. Castro MJL, Ojeda C, Cirelli AF. Advances in surfactants for agrochemicals. Environ Chem Lett. 2014;12(1):85-95. doi:10.1007/ s10311-013-0432-4

50. AOUADA, F. A.; DE MOURA MR. Nanotechnology applied in agriculture: Controlled release of agrochemicals. In: Rai M, Ribeiro C, Mattoso L, Duran N, eds. Nanotechnologies in Food and Agriculture. Cham: Springer International Publishing; 2015:103-118. doi:10.1007/978-3-319-14024-7

51. Desiraju GR. Crystal Engineering: A Holistic View. Angew Chemie Int Ed. 2007;46(44):8342-8356. doi:10.1002/anie.200700534

52. Dubey S, Jhelum V, Patanjali PK. Controlled release agrochemicals formulations: A review. J Sci Ind Res (India). 2011;70(2):105-112.

53. Jeschke P. Propesticides and their use as agrochemicals. Pest Manag Sci. 2016;72(2):210-225. doi:10.1002/ps.4170

54. Wouters J, Rome S, Quéré L. Monographs of most Frequent Co-Crystal Formers. In: Pharmaceutical Salts and Co-Crystals. RSC; 2011:338-382. doi:10.1039/9781849733502-00338

55. Wicker JGP, Crowley LM, Robshaw O, et al. Will they co-crystallize? CrystEngComm. 2017;(19):5336-5340. doi:10.1039/C7CE00587C

56. Zhou L, Dodd S, Capacci-Daniel C, Garad S, Panicucci R, Sethuraman V. Co-crystal formation based on structural matching. Eur J Pharm Sci. 2016;88:191-201. doi:10.1016/j.ejps.2016.02.017 
57. Kelley SP, Narita A, Holbrey JD, Green KD, Reichert WM, Rogers RD. Understanding the effects of ionicity in salts, solvates, co-crystals, ionic co-crystals, and ionic liquids, rather than nomenclature, is critical to understanding their behavior. Cryst Growth Des. 2013;13(3):965-975. doi:10.1021/cg4000439

58. Chen C, Liu F, Fan T, Peng Q. Improved solubility of sparingly soluble pesticides in mixed ionic liquids. RSC Adv. 2016;6(63):58106-58112. doi:10.1039/C6RA05012C

59. Braga D, Grepioni F, Chelazzi L, et al. Bentazon: Effect of Additives on the Crystallization of Pure and Mixed Polymorphic Forms of a Commercial Herbicide. Cryst Growth Des. 2014;14(11):5729-5736. doi:10.1021/cg500980j

60. Moss LE, Karcher BA, Richardson Jnr JW, Jacobson RA. Structure of 3-isopropyl-1H-2,1,3-benzothiadiazin-4(3H)-one 2,2-dioxide (bentazon). Acta Crystallogr Sect C Cryst Struct Commun. 1986;42(12):1785-1787. doi:10.1107/S010827018609056X

61. Bessegato GG, Santos VP, Lindino CA. Degradação fotoeletroquímica do herbicida bentazona sobre eletrodos de carbono modificados por TiO 2. Quim Nova. 2012;35(2):332-336. doi:10.1590/S010040422012000200019

62. Syngenta. Embrapa identifica resistência da lagarta-do-cartucho ao metomil.

63. Waite MG, Sim GA. Configuration of the S-alkyl thiohydroximates: crystal and molecular structures of syn-(alkylthio)-isomers of S-methyl and S-cyanoethyl O-(N-methylcarbamoyl)acetothiohydroximates. J Chem Soc B Phys Org. 1971:752. doi:10.1039/j29710000752

64. Takusagawa F, Jacobson RA. Crystal and Molecular Structure of Carbamate Insecticides. 3. Methomyl. J Agric Food Chem. 1977;25(3):577-581. doi:10.1021/jf60211a002

65. Aranda G, Gauvrit C, Cesario M, Guilhem J, Pascard C, Tran Huu Dau ME. Biological activity of the two geometrical isomers of methomyl on maize mitochondria. Phytochemistry. 1983;22(11):2431-2435. doi:10.1016/0031-9422(83)80134-4

66. Aliev AG, Atovmyan L 0., Kartsev VG. Structure of 4-amino-6tert-Butyl-3-methylthio- 1,2,4-triazin-5-one. J Struct Chem. 1989;30(6):1012-1013. doi:10.1007/BF00752809

67. Rossi CVS, Velini ED, Luchini LC, et al. Performance of metribuzin apllied on sugarcane straw | Dinâmica do herbicida metribuzin aplicado sobre palha de cana-de-açúcar (saccarum officinarum). Planta Daninha. 2013;31(1). doi:10.1590/S0100-83582013000100024

68. Lazaro SR de, Bortolini TJ, Oliveira CR de. Simulação computacional do herbicida Metribuzin. In: VI Workshop Da Rede de Nanotecnologia Aplicada Ao Agronegócio. Fortaleza: Embrapa Agroindústria Tropical; 2012:402-403.

69. Chopra D, Mohan TP, Rao KS, Row TNG. Exploring polymorphism by solvent mediation in potentially active herbicide Metribuzin: A subtle interplay of weak intermolecular interactions. CrystEngComm. 2005;7(62):374. doi:10.1039/b504347f

70. Franco MHR, França AC, Albuquerque MT, Schiavon NC, Vargas GN. Fitorremediação de solos contaminados com picloram por Urochloa brizantha. Pesq Agropec Trop. 2014;44(4):460-467.

71. Parthasarathi V, Wolfrum S, Noordik JH, et al. No Title. Cryst Struct Commun. 1982;(11):1519-1524.

72. Smith G. Crystal structure of poly[[di- $\mu 2$-aqua-aquasodium] 4-amino3,5,6-trichloropyridine-2-carboxylate trihydrate], the sodium salt of the herbicide picloram. Acta Crystallogr Sect E Crystallogr Commun. 2015;71(8):931-933. doi:10.1107/S2056989015012633

73. Gielen D, Boshell F, Saygin D. Climate and energy challenges for materials science. Nat Mater. 2016;15:117-120.

74. Ambrose A, Al-Amin A, Rasiah R, Saidur R, Amin N. Prospects for introducing hydrogen fuel cell vehicles in Malaysia. Int J Hydrogen Energy. 2017;42(14):9125-9134.

75. Erk $P$, Hengelsberg H, Haddow MF, van Gelder R. The innovative momentum of crystal engineering. CrystEngComm. 2004;6(78):474. doi:10.1039/b409282a

76. Torriani IL. Cristalografia: uma ciência multidisciplinar. Ciênciancia e Cult. 2014;66:4-5. http://cienciaecultura.bvs.br/scielo.php?script=sci arttext\&pid=S0009-67252014000300002\&nrm=iso.

\section{Agnaldo M. J. Junior ${ }^{1,2}$, Igor L. de Andrade2, Wesley F. Vaz 2,3*, Eduardo C. M. Faria ${ }^{2}$ \& Hamilton B. Napolitano', ${ }^{1,2}$}

\author{
${ }^{1}$ Centro Universitário de Anápolis, 75083-515, Anápolis, GO, Brasil \\ ${ }^{2}$ Universidade Estadual de Goiás, 75132-400, Anápolis, GO, Brasil \\ ${ }^{3}$ Instituto Federal de Educação, Ciência e Tecnologia de Mato Grosso, \\ 78455-00 Lucas do Rio Verde, MT, Brasil \\ "E-mail:wesfonseca@gmail.com
}

